\title{
Interaction between diet and sunlight exposure on vitamin $D$ status in premenopausal women living in Southern England: preliminary analysis of the Vitamin D, Food Intake, Nutrition and Exposure to Sunlight in Southern England (D-FINES) Study
}

\author{
A. L. Darling ${ }^{1}$, D. P. Lovell ${ }^{1}$, P. A. Lee ${ }^{1}$, W. T. K. Lee ${ }^{1}$, J. L. Berry ${ }^{2}$ and S. A. Lanham-New ${ }^{1}$ \\ ${ }^{1}$ Nutritional Sciences Division, Faculty of Health and Medical Sciences, University of Surrey, Guildford GU2 7HX, UK and \\ ${ }^{2}$ Vitamin D Research Group, Department of Medicine, University of Manchester, Manchester M13 9PL, UK
}

Vitamin D deficiency may increase the risk of osteoporosis, cancer, type 1 diabetes mellitus, and heart disease ${ }^{(1)}$. However, the relative contribution of UVB sunlight exposure and dietary vitamin D intake to vitamin D status is unclear. The aim of the present study was to examine these factors in combination using a hierarchical multiple regression model.

The 2006-7 D-FINES Study investigated 373 Surrey Caucasian and Asian women in four seasons of the year for vitamin D status, vitamin D dietary intake and UVB exposure. Dietary intake was assessed using $4 \mathrm{~d}$ estimated food diaries repeated every season and sunlight exposure by repeated wearing of UV badges (for 1 week) also in every season. The relative contribution of UVB exposure and dietary vitamin D intake to vitamin D status was assessed separately for Caucasian and Asian women because of the difficulty of separating effects of ethnicity from those of UV exposure and dietary intake of vitamin D. In the present preliminary analysis data on UVB exposure and vitamin D intake data from the subset of 135 Caucasian premenopausal women (mean age 33.8 (SD 5.6) years) were analysed.

For Caucasian premenopausal women, using hierarchical multiple regression analysis, the two variables of most interest were added first (UV and vitamin D intake), with other physiologically-relevant variables that showed a significant correlation with vitamin D status being added next. For UV exposure in step 1 the model explained $6.1 \%$ of variance in vitamin D status, a significant change (F change $P<0.05)$. With dietary intake of vitamin D added at step 2, a further $1.5 \%$ explanation of variance was added, although this change did not reach significance ( $F$ change $P>0.05$ ). In steps 3 and 4 factor of sunscreen used and total body fat mass were entered into the model, and added $3.4 \%$ and $2 \%$ of variance respectively ( $F$ change $P>0.05$ ). Ca intake and age were not entered into the model, as they were not found to be significantly correlated with vitamin $\mathrm{D}$ status in this data subset, as has been found in other studies ${ }^{(2)}$.

For the model as a whole the total variance in vitamin D status explained was $8.3 \%$ (adjusted $R^{2} 0.083 ; \mathrm{F} 4,73 ; P=0.035$ ). Standardised $\beta$ coefficients were 0.191 for UV, 0.121 for dietary intake of vitamin $\mathrm{D},-0.167$ for factor of sunscreen used and -0.147 for total fat mass. However, none of these four variables made a significant unique contribution $(P>0.05)$ to vitamin $\mathrm{D}$ status.

Overall, the regression model suggests that UVB, dietary intake, factor of sunscreen and total body fat mass make a small but significant contribution to determining vitamin D status, explaining approximately $8 \%$ of variance. Further analysis of the dataset is underway to determine the relative contribution of UVB exposure and dietary vitamin D intake to 25OHD status in Asian premenopausal women and the two post-menopausal Asian and Caucasian subgroups as well as determining, if possible, the specific unique contributions of each factor.

This work is funded by the FSA (Project no. NO5064). The kind help with subject recruitment given by the Islamic Resource Centre Kingston, Woking Khidmat Group, Woking Asian Women's Association, Thornton Health Asian Association and College Road Surgery, Woking is acknowledged.

1. Holick MF (2006) High prevalence of vitamin D inadequacy and implications for health. Mayo Clin Proc 81, 353-73.

2. Gilman J, Shanahan F \& Cashman KD (2006) Eur J Clin Nutr 60, 889-896. 